\title{
Decreased Neutrophil Extracellular Trap Degradation in Shiga Toxin-Associated Haemolytic Uraemic Syndrome
}

\author{
Jonatan Leffler ${ }^{a, b}$ Zoltán Prohászka ${ }^{c}$ Bálint Mikes ${ }^{c}$ György Sinkovits ${ }^{c}$ \\ Katarzyna Ciacma ${ }^{a}$ Péter Farkas ${ }^{c}$ Marienn Réti ${ }^{e} \quad$ Kata Kelen ${ }^{d}$ \\ György S. Reusz $^{d}$ Attila J. Szabód ${ }^{d}$ Myriam Martina Anna M. Blom ${ }^{a}$ \\ ${ }^{a}$ Section of Medical Protein Chemistry, Department of Translational Medicine, Lund University, Malmö, Sweden; \\ ${ }^{b}$ Telethon Kids Institute, University of Western Australia, Perth, W.A., Australia; ${ }^{\mathrm{C} T h i r d ~ D e p a r t m e n t ~ o f ~ M e d i c i n e ~ a n d ~}$

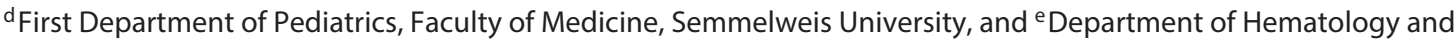 \\ Stem Cell Transplantation, St. István and St. László Hospital of Budapest, Budapest, Hungary
}

\section{Key Words}

Thrombotic microangiopathies · Neutrophil extracellular traps $\cdot$ Nuclease activity $\cdot$ Shiga toxin-associated haemolytic uraemic syndrome $\cdot$ Degradation $\cdot$ Kidney injury

\footnotetext{
Abstract

Background: Neutrophil extracellular traps (NETs) can stimulate thrombosis, and their degradation is decreased in several autoimmune disorders. It was recently reported that some patients with haemolytic uraemic syndrome (HUS) also fail to degrade NETs and that neutrophils from Shiga toxin-associated HUS are primed to form NETs. Method: We used a well-characterized cohort of 74 thrombotic microangiopathy (TMA) patients, with a subset also providing followup samples, and 112 age-matched controls to investigate NET degradation and serum nuclease activity in TMA before, during and after treatment. Results: We identified that in the cohort of TMA patients, $50 \%$ of patients with Shiga toxinassociated HUS displayed a decreased ability to degrade NETs. NET degradation correlated with serum nuclease activ-
}

ity, but not with autoantibodies against double-stranded DNA, which has been previously observed in some autoimmune disorders. Further, NET degradation negatively correlated with serum creatinine levels, suggesting that kidney function was negatively impacted by the low NET degradation ability. Conclusions: We revealed that decreased NET degradation is a common feature of Shiga toxin-associated HUS and that it is associated with decreased kidney function in these patients. It remains to be clarified whether improving NET degradation would be beneficial for the patient.

(c) 2016 S. Karger AG, Basel

\section{Introduction}

Thrombotic microangiopathies (TMA) such as thrombotic thrombocytopenic purpura (TTP) and haemolytic uraemic syndrome (HUS) involve thrombocytopenia,

J.L. and Z.P. contributed equally to this study.

\section{KARGER}

(c) 2016 S. Karger AG, Basel

E-Mail karger@karger.com

www.karger.com/jin
Dr. Anna M. Blom

Section of Medical Protein Chemistry, Department of Translational Medicine Malmö Lund University, Inga Marie Nilssons Street 53

SE-205 02 Malmö (Sweden)

E-Mail anna.blom@ med.lu.se 
anaemia and organ ischemia due to thrombi formation [1]. Mutations or autoantibodies against the metalloprotease ADAMTS13 are strongly associated with congenital and acquired TTP, respectively. HUS is mostly caused by Shiga toxin (Stx)-producing Escherichia coli (STEC) infections in young children [2]. STEC infections lead to acute bloody diarrhoea and abdominal cramps. Once Stx is released from the gut into the circulation, it is taken up through the Gb3 receptor by renal epithelial cells. Inside the cell, it causes cell death by inhibiting the ribosomal machinery leading to renal failure [3]. The pathology varies, but a large proportion (more than 50\%) of children with STEC require dialysis. Another form of HUS is atypical HUS (aHUS), which arises from dysregulation of the alternative complement pathway caused by either genetic alterations in complement proteins (autoantibody negative) or autoantibodies against these (autoantibody positive) [4]. In addition, other forms of TMA may also arise secondary to co-existing diseases, such as systemic autoimmune disease or cancer $[5,6]$. Current treatment options for TMAs include supportive care, electrolyte monitoring, plasma therapy and dialysis, as well as additional targeted therapies, such as complement inhibition therapy with eculizumab [7].

Elevated levels of circulating DNA have been reported in TMA patients [8], and we previously reported increased levels of neutrophil elastase in acute TTP, which is associated with disease activity markers of acute TTP [9]. These observations in combination with recent reports of a decreased degradation of neutrophil extracellular traps (NETs) in TMA [10] and increased formation of NETs [11] have suggested a role for NETs in thrombotic microangiopathic disorders. NETs consist of decondensed chromatin covered with antimicrobial proteins/peptides and are released from mainly activated neutrophils in a ROS/NADPH-dependent manner [12, 13]. NETs, while beneficial in antimicrobial responses, are also involved in a number of pathologies and in particular in autoimmune disorders such as systemic lupus erythematosus [14], where a decreased ability to degrade NETs [15], and other types of chromatin [16], is associated with kidney disease $[15,17]$. Given the kidney damage observed in TMA, we aimed to investigate whether this was associated with a decreased ability to degrade NETs, as has recently been suggested [10]. To achieve this, we used a well-characterized TMA cohort consisting of longitudinally collected samples from patients with various thrombotic microangiopathies.

\section{Materials and Methods}

\section{Patients and Sera}

Seventy-four patients with TMA (24 men and 50 women) with a median age of 24.5 years (range 15 days to 74 years) and 112 controls (67 women and 45 women) with a median age of 34 years (range 5-59 years) were enrolled into this study. Samples were selected from patients referred for complement and ADAMTS13 testing for diagnostic purposes to the Research Laboratory at the 3rd Department of Medicine (Semmelweis University, Budapest, Hungary) between 2008 and 2013. Active TMA was defined as thrombocytopenia $(<150 \mathrm{~g} / \mathrm{l})$, direct Coombs testnegative haemolysis with fragmentocytes in the peripheral blood smear and elevated lactate dehydrogenase (LDH) levels $(>450$ $\mathrm{U} / \mathrm{l}$ ) with the presence of organ involvement of various extents. STEC-HUS was diagnosed when STEC infection could be verified. Secondary TMA was defined when evidence of a coexisting disease or treatment was established, such as malignancy $(\mathrm{n}=3)$, autoimmune disease $(n=2)$, sepsis $(n=4)$ and non-renal transplantation $(n=8)$. aHUS was present when ADAMTS13 deficiency and STEC infection were excluded and a co-existing disease was not diagnosed. The subgroup of anti-factor $\mathrm{H}$ autoantibody-positive aHUS patients is shown separately. For the STEC-HUS group, samples in the acute phase were received within 7 days after the start of bloody diarrhoea. All patients were discharged when remission was reached, i.e. if haematologic activity of TMA was not present (platelet numbers $>150 \mathrm{~g} / \mathrm{l}$ on 2 consecutive days) and patients no longer required a plasma exchange. Follow-up (control) samples of STEC-HUS patients were taken at least 7 days after discharge but typically after a mean of 38 days, with a SD of 23 days. Healthy controls were recruited from an adult outpatient department of occupational health or among children waiting for minor elective surgical interventions in the same time period. Subjects without a known disease or regular medical treatment, except for adequately controlled hypertension, were enrolled into the control group. Baseline clinical and laboratory characteristics are described in table 1 . Samples were taken from antecubital venipuncture or from a central venous catheter. The cells and supernatant were separated by centrifugation and shipped in a cooled package to our lab. Separated samples were stored at $<70^{\circ} \mathrm{C}$ until use. All patients and healthy controls gave informed consent to participate in this study, which was approved by the highest ethics committee (ETT TUKEB, Budapest, Hungary). This study was conducted according to the Helsinki Declaration.

\section{Isolation of Neutrophils}

Neutrophils were isolated from the blood of healthy volunteers according to a previously published method [18]. Briefly, blood was separated by centrifugation on a Histopaque-1119 column (Sigma-Aldrich), and the granulocyte-rich fraction was isolated and washed; neutrophils were isolated by centrifugation on a Percoll gradient (65-80\%) (GE Healthcare) and isolated from the intersection of the 70 and $75 \%$ layer, washed and resuspended in RPMI with 10 mM Hepes buffer. The purity of neutrophils (>80\%) was determined by flow cytometry (CyFlow Space; Partec) for surface marker expression for anti-CD14 (BD), anti-CD15 and antiCD16 (both from ImmunoTools). Neutrophils were defined as $\mathrm{CD} 16^{+} / \mathrm{CD} 15^{+} / \mathrm{CD} 14^{\text {low }}$ and the data were evaluated using FlowJo software (TreeStar). 
Table 1. Characteristics of the study participants

\begin{tabular}{llll}
\hline Variable & $\begin{array}{l}\text { TMA patients (excluding } \\
\text { STEC-HUS }(\mathrm{n}=44)^{\mathrm{a}}\end{array}$ & $\begin{array}{l}\text { STEC-HUS } \\
(\mathrm{n}=30)^{\mathrm{a}}\end{array}$ & $\begin{array}{l}\text { Controls } \\
(\mathrm{n}=112)\end{array}$ \\
\hline $\begin{array}{l}\text { Age, years } \\
\text { Female/male ratio }\end{array}$ & $38(22-55)$ & $4(1-69)$ & $34(27-41)$ \\
Subgroup & $28 / 16$ & $22 / 8$ & $67 / 45$ \\
$\quad$ & 17 & & \\
$\quad$ ADAMTS13-deficient TTP & 9 & & None \\
$\quad$ aHUS & 18 & $0 / 30 / 0 / 0$ & $69(62-83)$ \\
$\quad$ Secondary TMA & $20 / 20 / 3 / 1$ & $286.5(38-862)$ & $138(128-149)$ \\
Kidney injury (none/acute only/ & $85(63.75-136.75)$ & $91(61-136)$ & $276(25-317)$ \\
$\quad$ acute on chronic/chronic only), $\mathrm{n}$ & $85(70.5-97.5)$ & $43(13-436)$ & N/A \\
Creatinine, $\mu$ mol/l & $41(23-73.5)$ & $2,021(54-6,348)$ & \\
Haemoglobin, g/l & $1,791(672-3,036)$ & & \\
Platelets, g/l & & & \\
Lactate dehydrogenase activity, U/l & &
\end{tabular}

Data represent medians (IQR) or numbers of subjects unless otherwise stated. N/A = Not available.

${ }^{a}$ With the available sample for NET degradation analysis taken before the initiation of plasma therapy.

Generation and Degradation of NETs

Freshly isolated neutrophils (50,000 cells/sample) were seeded onto a 96-well flat-bottom plate (Nunc) and incubated with $20 \mathrm{nM}$ PMA (Sigma-Aldrich) for $4 \mathrm{~h}$ at $37^{\circ} \mathrm{C}$ and $5 \% \mathrm{CO}_{2}$ to generate NETs according to a previously published method $[15,16,19]$. NET formation was confirmed visually by staining NETs with propidium iodide (Molecular Probes) and analysed using a Zeiss LSM 510 Meta confocal microscope (Zeiss). The cell medium was removed from NETs and $5-10 \%$ patient or control sera, as indicated, in $10 \mathrm{mM}$ Tris- $\mathrm{HCl}$ (pH 7.5), $50 \mathrm{mM} \mathrm{NaCl}, 10 \mathrm{mM} \mathrm{MgCl}_{2}$ and $2 \mathrm{mM}$ $\mathrm{CaCl}_{2}$ (DNase buffer) was added with or without $2 \mathrm{mU}$ recombinant human DNase-I (Bioworld) or $2 \mathrm{mU}$ micrococcal nuclease (MNase; Worthington Biochemical Corporation) and incubated for $60 \mathrm{~min}$ at $37^{\circ} \mathrm{C}$. During this time, the degraded NETs were released into the solution. An aliquot of the NET-containing solution was then transferred to PBS with a final concentration of 2 mM EDTA to stop further degradation. The DNA content of supernatants from the NET degradation assay or diluted serum samples was quantified using PicoGreen (Invitrogen). The DNA quantity ranged from 50 to $2,700 \mathrm{ng} / \mathrm{ml}$ and was within the linear range $(5-5,000 \mathrm{ng} / \mathrm{ml})$ of the standard curve. As an internal control, pooled normal human serum (NHS) was used and all samples were compared to the mean of the internal controls for each individual experiment. All samples were measured in duplicate and the mean was used for analysis.

\section{Nuclease Zymogram}

Sera from patients and controls were diluted in DNase buffer to $30 \%$ and loaded onto a native PAGE gel containing $22 \mu \mathrm{g} / \mathrm{ml}$ denatured calf thymus DNA (Sigma-Aldrich). As an internal control, 30\% NHS was used. There was a linear relationship between the amount of serum loaded onto the gel and the nuclease activity detected in our readout. Gels were run at $90 \mathrm{~V}$ for $2 \mathrm{~h}$ under native non-reducing conditions, washed with $\mathrm{dH}_{2} \mathrm{O}$ and incubated with $40 \mathrm{~mm}$ Tris- $\mathrm{HCl}$ ( $\mathrm{pH} 7.5$ ), $8 \mathrm{mM} \mathrm{MgCl}_{2}, 2 \mathrm{~mm} \mathrm{CaCl}_{2}$ and $0.02 \%$
$\mathrm{NaN}_{3}$ at $37^{\circ} \mathrm{C}$ for $18 \mathrm{~h}$. After this, ethidium bromide was added to a final concentration of $1 \mu \mathrm{g} / \mathrm{ml}$ and gels were incubated at $37^{\circ} \mathrm{C}$ for an additional $30 \mathrm{~min}$ and analysed in a ChemiDoc ${ }^{\mathrm{TM} M P}$ Imaging System (BioRad).

\section{Determination of Additional Laboratory Parameters}

Baseline clinical laboratory parameters (creatinine and LDH) were measured using a Roche Integra 800 analyser (Roche), and the complete blood count was obtained using a Cell-Dyn 3500 haematology analyser (Abbott). The methods for the determination of ADAMTS13 activity, complement factors, complement inhibitors, autoantibodies and genetic investigation have been described earlier [20]. Levels of anti-double-stranded DNA autoantibodies (antidsDNA) were determined using an AUTOSTAT II anti-dsDNA ELISA (Hycor, USA) according to the manufacturer's instructions. Levels of anti-dsDNA were expressed in international unit per millilitre, and the cutoff values provided by the manufacturer were the following: <40 IU/ml, negative; 40-60 IU/ml, equivocal, and $>60$, positive.

\section{Statistical Analyses}

Since most continuous variables in this study showed a skewed distribution, and failed the Shapiro-Wilk's test, a non-parametric approach was employed for all analysis. For comparison of NET degradation, DNase activity and anti-dsDNA levels between two groups, the Mann-Whitney U test was used. For comparison between multiple clinical groups or time points, the Kruskal-Wallis test followed by Dunn's multiple-comparisons test was used. To test differences in proportions Pearson's $\chi^{2}$ test was used, and to test the correlation between continuous variables Spearman's correlation test was used. Since the longitudinal sample set was not complete, we did not use a paired analysis. We used both GraphPad Prism 5 (GraphPad) and JMP 11 (SAS) for statistical analyses of experimental and clinical data, respectively. Two-tailed $p$ values were calculated, and the significance level was set at 0.05 . 

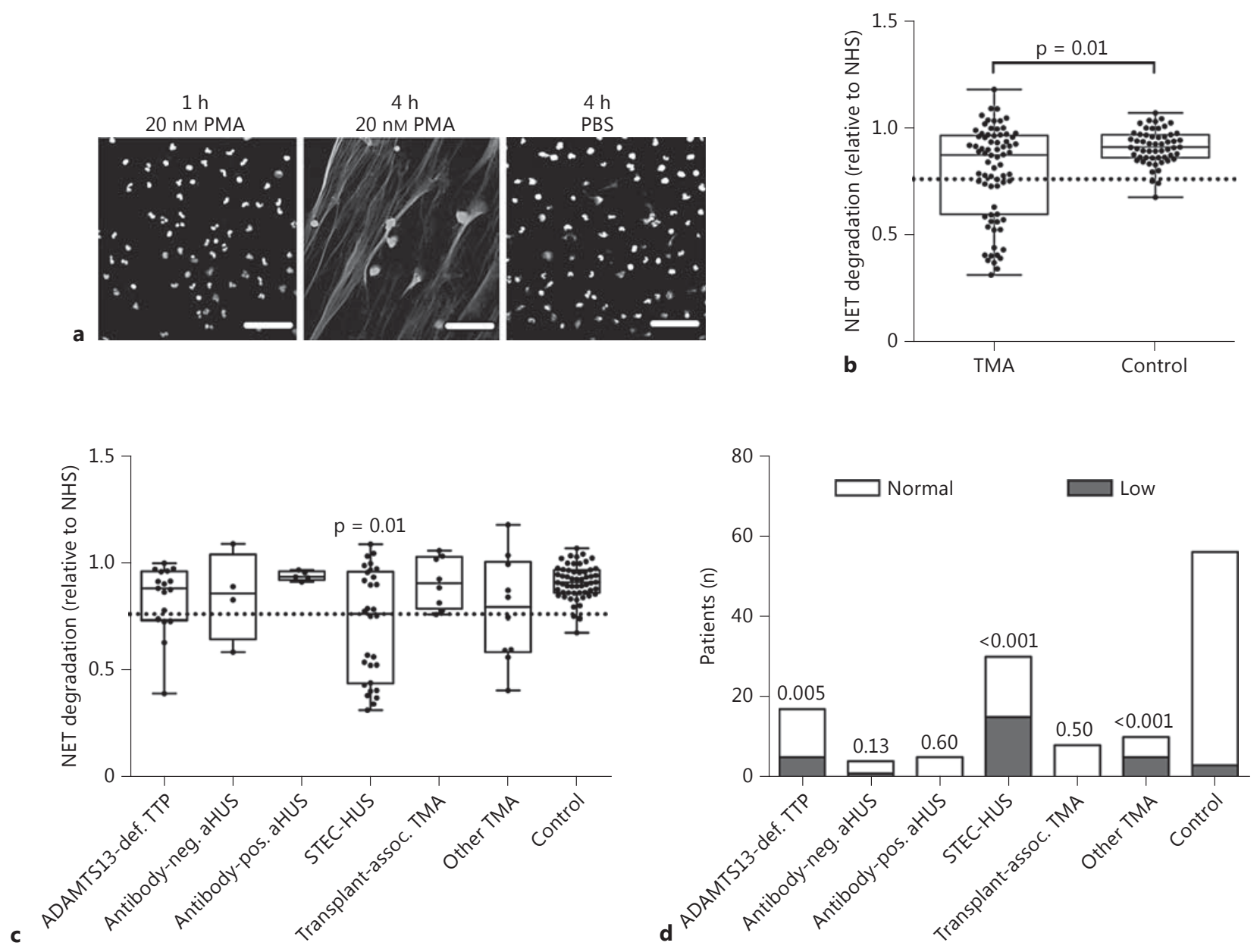

Fig. 1. a Neutrophils were stimulated with PMA to produce NETs. NETs were formed after $4 \mathrm{~h}$, while no NETs were formed without stimulation. b Degradation of NETs by $10 \%$ serum from TMA patients with acute disease $(n=74)$ compared to healthy controls $(n=56)$. Patients with degradation $<2$ SD below the mean degradation of the healthy control group were defined as 'low', and this cutoff is indicated by the dotted line. Degradation of NETs (c) or percentage of low-degrading patients (d) in subgroups of TMA patients with ADAMTS13-deficient TTP $(\mathrm{n}=17)$, anti-factor $\mathrm{H}$ positive $(n=5)$ or -negative $(n=4)$ aHUS, STEC-HUS $(n=30)$, transplant-associated TMA $(n=8)$ or other forms of TMA such as secondary to infectious or malignant diseases $(n=10)$. The significance of differences between 2 groups was calculated using a Mann-Whitney U test, and for multiple groups a Kruskal-Wallis test followed by Dunn's multiple-comparisons test was used. For proportion data, the significance of differences compared to controls was calculated using Pearson's $\chi^{2}$ test. $p$ values above each group indicate the statistical difference compared to healthy controls. Scale bars $=50 \mu \mathrm{M}$. def. $=$ Deficient; neg. = negative; pos. $=$ positive; assoc. $=$ associated.

\section{Results}

\section{Patients with STEC-HUS Have a Decreased Ability to} Degrade NETs

A decreased ability to degrade DNA in the form of NETs is associated with kidney disease in systemic lupus erythematosus $[15,17]$ and was recently suggested to be involved in TMA pathology [10]. In the present study, NETs were induced by PMA stimulation of neutrophils isolated from healthy volunteers (fig. 1a). To investigate the role of serum nuclease activity in TMA in an independent and well-characterized cohort, we analysed NET degradation in patients with acute TMA before any treatment (fig. 1). We defined NET degradation below 2 SD of 

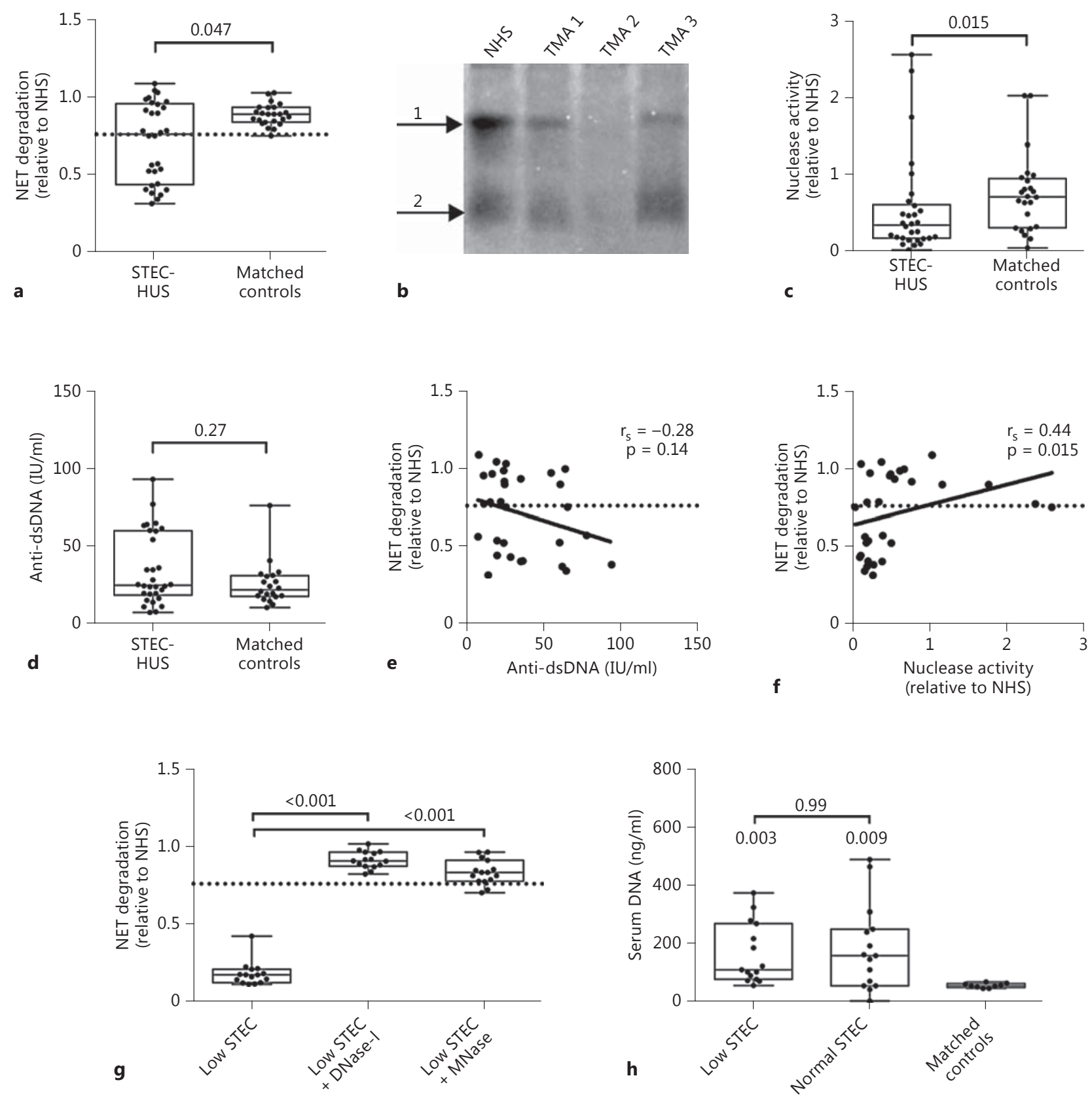

Fig. 2. Degradation of NETs (a) and total nuclease activity (b, c) by $10 \%$ serum from patients with acute STEC-HUS $(n=30)$ compared to matched healthy controls $(n=24)$. $\mathbf{b}$ Representative DNA zymogram depicting the nuclease activity in TMA patients 1-3 and the internal control (NHS). Nuclease activity is found in two forms in serum, i.e. free DNase-I (arrow 2) and DNase-I in complex with a serum protein (arrow 1). d Titres of anti-dsDNA antibodies in STEC-HUS patients with acute disease $(\mathrm{n}=30)$ compared to age-matched healthy controls $(n=20)$. e, $\mathbf{f}$ Correlation between NET degradation, anti-dsDNA titres and nuclease activity in STEC-HUS patients with acute disease. $\mathbf{g}$ NET degradation by $5 \%$ serum from low-degrading STEC-HUS patients $(n=15)$ compared to serum supplemented with $2 \mathrm{mU}$ DNase-I or $2 \mathrm{mU}$ MNase. $\mathbf{h}$ Levels of serum DNA in STEC-HUS patients with low $(\mathrm{n}=15)$ and normal $(\mathrm{n}=15)$ NET degradation and matched controls $(n=9)$. The significance of differences between groups, compared to healthy controls, was calculated using a Mann-Whitney U test, and for multiple groups a Kruskal-Wallis test followed by Dunn's multiple-comparisons test was used. Correlations were calculated using a non-parametric Spearman's correlation test. $\mathrm{p}$ values above each group indicate the statistical difference compared to healthy controls. 
the mean degradation of the healthy control group as 'low'. We observed that a subset of patients with acute TMA had a significantly decreased ability to degrade NETs compared to healthy controls (fig. 1b). To identify whether this subset of patients represented a particular type of TMA, we analysed the degradation in clinical subsets of TTP and HUS patients (fig. 1c). Interestingly, only patients with STEC-HUS displayed a significant decrease in NET degradation as a group compared to healthy controls. When analysing the percentage of patients with low degradation in each TMA subset, we observed that $29 \%$ of patients with ADAMTS13-deficient TTP, $50 \%$ of patients with STEC-HUS and 50\% of patients with other forms of TMA displayed a significantly different proportion of low degradation compared to controls (5\%; fig. 1d). Since the TMA subset of ADAMTS13-deficient TTP and other forms of TMA was relatively small ( $\mathrm{n}<$ 20 ), we focused the remainder of this study on the STECHUS patients $(n=30)$.

Most patients with STEC-HUS are young (median age 5 years) and we therefore only included controls of a similar age (median age 15 years) for the subsequent analysis. Since no association with age was observed for NET degradation, nuclease activity or anti-dsDNA levels in our cohort (data not shown), the slightly higher median age of our controls should not have affected the analysis. In patients with STEC-HUS the ability to degrade NETs was significantly lower compared to that of matched controls (fig. 2a). To determine whether this was due to a decreased serum nuclease activity, as some nucleases are produced in the kidneys [21], we analysed nuclease activity using a native DNA zymogram (fig. 2b, c). STEC-HUS patients had a significantly decreased serum nuclease activity compared to controls, which can affect NET degradation. A decreased ability to degrade NETs has previously been associated with the presence of autoantibodies against dsDNA that sterically hinder the access of nucleases and thus the degradation of NETs [15]. We therefore analysed the levels of dsDNA antibody in these patients without detecting any significant differences (fig. 2d). In systemic lupus erythematosus, there is a clear correlation between NET degradation and levels of anti-dsDNA [15, 19]. However, in the current dataset we did not observe any correlation with NET degradation and anti-dsDNA in STEC-HUS patients (fig. 2e). We did, however, observe a significant correlation between NET degradation and nuclease activity (fig. 2f), indicating that a low nuclease activity is associated with a decreased ability to degrade NETs. Of note is that nuclease activity did not correlate with anti-dsDNA titres (data not shown). To elucidate whether DNase-I specific inhibitors prevented NET degradation in the low-degrading STEC patients, we repeated the assay in the presence of human DNase-I or bacterial MNase. We observed that both nucleases restored degradation (fig. $2 \mathrm{~g}$ ), suggesting that the decreased activity is reversible. Elevated levels of serum DNA might potentially disturb the assay and be the reason for the decreased nuclease activity. Thus, we determined the serum DNA level in STEC patients and indeed observed significantly higher levels compared to healthy controls (fig. $2 \mathrm{~h}$ ). However, there was no difference in serum DNA levels between STEC patients with a low and normal ability to degrade NETs. Furthermore, the assay was not affected by the addition of plasmid DNA to NHS at concentrations exceeding the DNA concentration found in STEC patient sera (data not shown).

\section{The Ability to Degrade NETs Changes during}

\section{Treatment in TMA Patients}

In addition to serum samples taken before treatment, we also analysed the capacity of STEC-HUS patient sera to degrade NETs in remission. Not all patients were sampled at all time points, but lines connect samples from the same patient (fig. 3). We did not observe a statistically significant increase in NET degradation between the acute samples and samples taken during remission (fig. 3a), although $75 \%$ of the paired samples showed an increase in degradation. For the whole TMA cohort, we did observe a significant decrease in degradation from samples taken during treatment compared to healthy controls (fig. 3b). This suggests that, although the ability to degrade NETs may be normal upon hospital admission, some patients experience a temporary decrease in NET degradation as part of their TMA pathology. In addition, no difference in nuclease activity or titres of antidsDNA was observed between acute disease and remission in STEC-HUS patients (fig. 3c, d).

\section{A Decreased Ability to Degrade NETs Is Associated} with Impaired Kidney Function in STEC-HUS

A decreased ability to degrade NETs has been associated with clinical manifestations, including kidney injury, in multiple diseases $[15,17,22]$. We therefore evaluated the clinical impact of a decreased ability to degrade NETs in STEC-HUS patients. A high serum level of creatinine is a strong marker for decreased kidney function and in patients with STEC-HUS. We observed that the ability to degrade NETs is negatively associated with serum creatinine levels in the same sample (fig. $4 \mathrm{a}$ ), indicating decreased kidney function. On the other hand, we did not 
Fig. 3. Degradation of NETs in STEC-HUS patients before treatment $(n=30)$ and in remission $(n=9)(\mathbf{a})$, in TMA patients before treatment $(n=74)$, during treatment $(\mathrm{n}=23)$ and in remission $(\mathrm{n}=48)(\mathbf{b})$ and in matched healthy controls $(\mathrm{n}=24)$. Nuclease activity (c) and anti-dsDNA titres (d) in STEC-HUS patients before treatment and in remission compared to healthy controls. Multiple time points from the same patients are connected by a line (STEC-HUS, $\mathrm{n}=8$; TMA, $\mathrm{n}=26$ ). The significance of differences was calculated using a Kruskal-Wallis test with Dunn's posttest and is indicated by $\mathrm{p}$ values.

Fig. 4. Correlation between NET degradation and serum creatinine levels (a), platelet count (b), serum lactate dehydrogenase (c) and blood urea nitrogen (d) in patients with STEC-HUS $(n=30)$. The shaded areas indicate the normal ranges. Correlation was calculated using a non-parametric Spearman's correlation test $\left(\mathrm{r}_{\mathrm{S}}\right)$ and $\mathrm{p}$ values indicate the significance of the correlation.
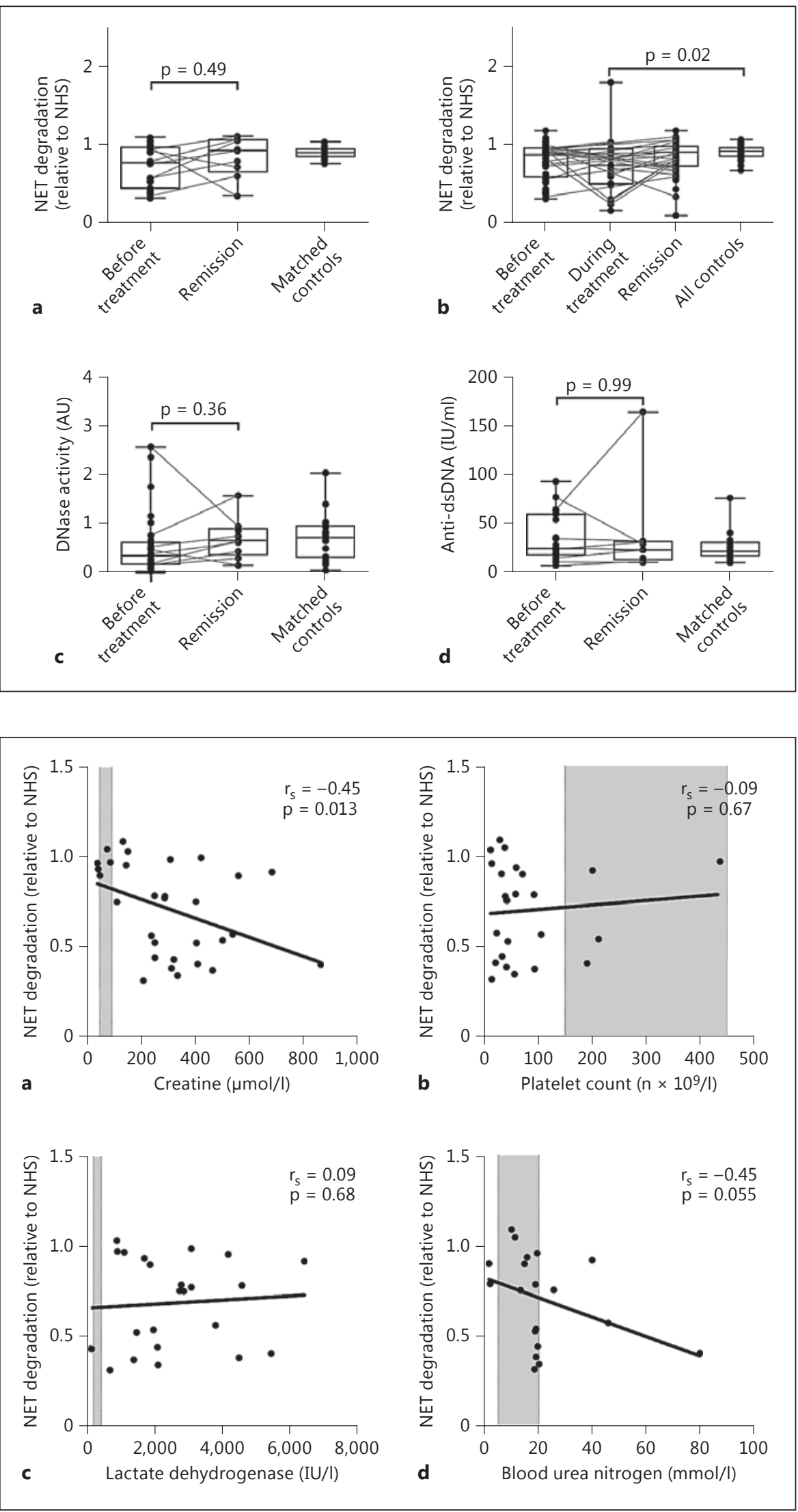
detect any significant association with other STEC-HUSassociated manifestations such as platelet count, serum levels of LDH and blood urea nitrogen (fig. $4 \mathrm{~b}-\mathrm{d}$ ).

\section{Discussion}

In the present study, we investigate the ability of patients with TMA to degrade NETs and report that in particular patients with STEC-HUS have a decreased ability to degrade NETs. Interestingly, this is not associated with elevated levels of anti-dsDNA titres as observed in other autoimmune diseases $[15,19]$ but rather with decreased nuclease activity. Decreased NET degradation was associated with disease activity in the TMA cohort but did not significantly differ between acute disease and remission, after a mean of 38 days, in STEC-HUS patients. However, recovery after a TMA episode is slow and a longer followup time might be preferable in future studies.

NETs were initially described as an innate defence mechanism against extracellular or severe microbial infections [12]. The discovery of NETs provided an explanation for observations that many microbes produce nucleases, but it also implied that the body may have mechanisms that prevent endogenous accumulation of NETs by mainly serum DNase-I [15] and that these can be regulated during infection. DNase- $\mathrm{I}$ is a $\mathrm{Ca}^{2+}$ - and $\mathrm{Mg}^{2+}$-dependent enzyme [21] that degrades extracellular DNA, chromatin exposed by dying cells and DNA in NETs. Globular actin in the presence of ATP can inhibit DNase-I [23], potentially presenting a mechanism for how tissue damage that releases ATP and actin can prevent NET degradation during infections. It was recently shown that neutrophils from Stx-associated HUS patients are more likely to produce NETs [11], and it is therefore tempting to speculate that the decreased NET degradation which we observed in STEC-HUS may be part of the acute response to fight the infection. As a consequence, increased NET release in combination with attenuated degradation may adversely contribute to some of the kidney damage in STEC-HUS through STEC-independent mechanisms such as the pro-thrombotic properties of NETs [24]. NETs can serve as scaffolding that traps platelets [25] and express the pro-thrombotic tissue factor that initiates coagulation [26, 27]. We and others have also previously reported that NETs can activate complement $[19,28]$ and this could enhance the Stx-induced complement activation leading to $\mathrm{C} 3 \mathrm{~b}$ deposition in the glomeruli and on platelets $[29,30]$, inducing microvascular thrombosis and kidney injury [31]. These adverse pro- coagulant, pro-inflammatory and complement-activating effects of NETs may be particularly severe in kidneys and fit well the known pathology of STEC-HUS. This novel subset of STEC-HUS patients characterized by impaired NET degradation does not differ in any routine clinical parameter except for creatinine levels. This is intriguing and could indicate a novel disease subset with specific pathophysiological mechanisms related to decreased nuclease activity. Unfortunately, we do not have data available on the serotype of pathogen causing the infection to rule out direct effects of specific pathogens and potential released virulence factors on NET degradation. One additional hypothesis explaining our findings is that STEC-HUS leads to impairment of kidney function, which translates into decreased DNase-I synthesis and levels in blood as in a mouse model of nephritis [32]. That in turn causes decreased NET degradation, which further damages the kidneys. We observed that STECHUS patients in general have higher levels of serum DNA compared to controls. This suggests a decreased ability to degrade chromatin released from dying cells, also in vivo, although this did not appear to be dependent on NETdegrading abilities. Unfortunately, we have not been able to directly verify decreased levels of DNase-I since there is no reliable immune assay measuring the DNase-I concentration in blood [10]. Upon addition of human recombinant DNase-I or microbial MNase to patient serum, NET degradation could be restored, suggesting that: (i) DNase-I is not blocked by an inhibitor with a high affinity or in large molar excess of DNase-I and (ii) the decreased NET degradation may be restored using DNase-I administered as therapy to these patients. Given the beneficial effects of NETs in fighting pathogens, it remains to be elucidated whether treatment with nucleases to minimize kidney damage would lead to a hampered defence against infection or indeed improve kidney function. Further studies are necessary to investigate the causes and consequences of decreased NET degradation in TMA and to determine whether drugs that inhibit NET formation or facilitate NET degradation may be used to treat STECHUS.

In summary, we found that $50 \%$ of patients with STECHUS failed to degrade NETs. This is associated with decreased kidney function and may provide clues for variation in disease pathology. Assessment of NET degradation may hence be useful as a biomarker for disease activity. In addition, treatment of these patients with exogenous nucleases that do not exacerbate infection may provide a novel future therapeutic strategy. 


\section{Acknowledgements}

The author would like to acknowledge all of the patients and their physicians as well as the following funding bodies: the Swedish Research Council (K2012-66X-14928-09-5), the Foundations of Österlund, Greta and Johan Kock, King Gustav V's 80th Anni- versary, Knut and Alice Wallenberg, Inga-Britt and Arne Lundberg, Torsten Söderberg and the Royal Physiographic Society in Lund. This study was also supported by grants for clinical research (ALF and the Skåne University Hospital) and by the Hungarian National Research Fund (OTKA 100687 and 108688).

\section{References}

1 Shenkman B, Einav Y: Thrombotic thrombocytopenic purpura and other thrombotic microangiopathic hemolytic anemias: diagnosis and classification. Autoimmun Rev 2014;13:584-586.

2 Scheiring J, Andreoli SP, Zimmerhackl LB: Treatment and outcome of Shiga-toxin-associated hemolytic uremic syndrome (HUS) Pediatr Nephrol 2008;23:1749-1760.

3 Sandvig K: Shiga toxins. Toxicon 2001;39: 1629-1635.

4 Nester CM, Barbour T, de Cordoba SR, Dragon-Durey MA, Fremeaux-Bacchi V, Goodship TH, Kavanagh D, Noris M, Pickering M, Sanchez-Corral P, Skerka C, Zipfel P, Smith RJ: Atypical aHUS: state of the art. Mol Immunol 2015;67:31-42.

5 George JN, Charania RS: Evaluation of patients with microangiopathic hemolytic anemia and thrombocytopenia. Semin Thromb Hemost 2013;39:153-160.

6 George JN, Terrell DR, Vesely SK, Kremer Hovinga JA, Lammle B: Thrombotic microangiopathic syndromes associated with drugs, HIV infection, hematopoietic stem cell transplantation and cancer. Presse Med 2012;41:e177-e188.

7 Scully M, Goodship T: How I treat thrombotic thrombocytopenic purpura and atypical haemolytic uraemic syndrome. Br J Haematol 2014;164:759-766.

8 Fuchs TA, Kremer Hovinga JA, Schatzberg D, Wagner DD, Lammle B: Circulating DNA and myeloperoxidase indicate disease activity in patients with thrombotic microangiopathies. Blood 2012;120:1157-1164.

9 Mikes B, Sinkovits G, Farkas P, Csuka D, Schlammadinger A, Razso K, Demeter J, Domjan G, Reti M, Prohaszka Z: Elevated plasma neutrophil elastase concentration is associated with disease activity in patients with thrombotic thrombocytopenic purpura. Thromb Res 2014;133:616-621.

10 Jimenez-Alcazar M, Napirei M, Panda R, Kohler EC, Kremer Hovinga JA, Mannherz HG, Peine S, Renne T, Lammle B, Fuchs TA Impaired DNase1-mediated degradation of neutrophil extracellular traps is associated with acute thrombotic microangiopathies. J Thromb Haemost 2015;13:732-742.
11 Ramos MV, Mejias MP, Sabbione F, Fernandez-Brando RJ, Santiago AP, Amaral MM, Exeni R, Trevani AS, Palermo MS: Induction of neutrophil extracellular traps in Shiga toxin-associated hemolytic uremic syndrome. J Innate Immun 2016;8:400-411.

12 Brinkmann V, Reichard U, Goosmann C, Fauler B, Uhlemann Y, Weiss DS, Weinrauch Y, Zychlinsky A: Neutrophil extracellular traps kill bacteria. Science 2004;303: 1532-1535.

13 Bjornsdottir $\mathrm{H}$, Welin A, Michaelsson E, Osla V, Berg S, Christenson K, Sundqvist M, Dahlgren C, Karlsson A, Bylund J: Neutrophil net formation is regulated from the inside by myeloperoxidase-processed reactive oxygen species. Free Radic Biol Med 2015;89: 1024-1035.

14 Knight JS, Kaplan MJ: Lupus neutrophils: 'NET' gain in understanding lupus pathogenesis. Curr Opin Rheumatol 2012;24:441450 .

15 Hakkim A, Furnrohr BG, Amann K, Laube B, Abed UA, Brinkmann V, Herrmann M, Voll RE, Zychlinsky A: Impairment of neutrophil extracellular trap degradation is associated with lupus nephritis. Proc Natl Acad Sci USA 2010;107:9813-9818.

16 Leffler J, Ciacma K, Gullstrand B, Bengtsson AA, Martin M, Blom AM: A subset of patients with systemic lupus erythematosus fails to degrade DNA from multiple clinically relevant sources. Arthritis Res Ther 2015; $17: 205$

17 Villanueva E, Yalavarthi S, Berthier CC, Hodgin JB, Khandpur R, Lin AM, Rubin CJ, Zhao W, Olsen SH, Klinker M, Shealy D, Denny MF, Plumas J, Chaperot L, Kretzler M, Bruce AT, Kaplan MJ: Netting neutrophils induce endothelial damage, infiltrate tissues, and expose immunostimulatory molecules in systemic lupus erythematosus. J Immunol 2011;187:538-552.

18 Brinkmann V, Laube B, Abu Abed U, Goosmann C, Zychlinsky A: Neutrophil extracellular traps: how to generate and visualize them. J Vis Exp 2010:e1724.
19 Leffler J, Martin M, Gullstrand B, Tyden H, Lood C, Truedsson L, Bengtsson AA, Blom AM: Neutrophil extracellular traps that are not degraded in systemic lupus erythematosus activate complement exacerbating the disease. J Immunol 2012;188:3522-3531.

20 Szarvas N, Szilagyi A, Csuka D, Takacs B, Rusai K, Muller T, Arbeiter K, Reti M, Haris A, Wagner L, Torok S, Kelen K, Szabo AJ, Reusz GS, Morgan BP, Prohaszka Z: Genetic analysis and functional characterization of novel mutations in a series of patients with atypical hemolytic uremic syndrome. Mol Immunol 2016;71:10-22.

21 Shiokawa D, Tanuma S: Characterization of human DNase I family endonucleases and activation of DNase gamma during apoptosis. Biochemistry 2001;40:143-152.

22 Yoshida M, Sasaki M, Sugisaki K, Yamaguchi Y, Yamada M: Neutrophil extracellular trap components in fibrinoid necrosis of the kidney with myeloperoxidase-ANCA-associated vasculitis. Clin Kidney J 2013;6:308-312.

23 Lazarides E, Lindberg U: Actin is the naturally occurring inhibitor of deoxyribonuclease I. Proc Natl Acad Sci USA 1974;71:47424746.

24 Fuchs TA, Brill A, Wagner DD: Neutrophil extracellular trap (NET) impact on deep vein thrombosis. Arterioscler Thromb Vasc Biol 2012;32:1777-1783.

25 Clark SR, Ma AC, Tavener SA, McDonald B, Goodarzi Z, Kelly MM, Patel KD, Chakrabarti S, McAvoy E, Sinclair GD, Keys EM, Allen-Vercoe E, Devinney R, Doig CJ, Green FH, Kubes P: Platelet TLR4 activates neutrophil extracellular traps to ensnare bacteria in septic blood. Nat Med 2007;13:463-469.

26 von Bruhl ML, Stark K, Steinhart A, Chandraratne S, Konrad I, Lorenz M, Khandoga A, Tirniceriu A, Coletti R, Kollnberger M, Byrne RA, Laitinen I, Walch A, Brill A, Pfeiler S, Manukyan D, Braun S, Lange P, Riegger J, Ware J, Eckart A, Haidari S, Rudelius M, Schulz C, Echtler K, Brinkmann V, Schwaiger M, Preissner KT, Wagner DD, Mackman N, Engelmann B, Massberg S: Monocytes, neutrophils, and platelets cooperate to initiate and propagate venous thrombosis in mice in vivo. J Exp Med 2012; 209:819-835 
27 Kambas K, Mitroulis I, Apostolidou E, Girod A, Chrysanthopoulou A, Pneumatikos I, Skendros P, Kourtzelis I, Koffa M, Kotsianidis I, Ritis K: Autophagy mediates the delivery of thrombogenic tissue factor to neutrophil extracellular traps in human sepsis. PLoS One 2012;7:e45427.

28 Wang $\mathrm{H}$, Wang C, Zhao $\mathrm{MH}$, Chen M: Neutrophil extracellular traps can activate alternative complement pathways. Clin Exp Immunol 2015;181:518-527.
29 Morigi M, Galbusera M, Gastoldi S, Locatelli M, Buelli S, Pezzotta A, Pagani C, Noris M, Gobbi M, Stravalaci M, Rottoli D, Tedesco F, Remuzzi G, Zoja C: Alternative pathway activation of complement by Shiga toxin promotes exuberant $\mathrm{C} 3 \mathrm{a}$ formation that triggers microvascular thrombosis. J Immunol 2011; 187:172-180.

30 Stahl AL, Sartz L, Karpman D: Complement activation on platelet-leukocyte complexes and microparticles in enterohemorrhagic Escherichia coli-induced hemolytic uremic syndrome. Blood 2011;117:5503-5513.
31 Locatelli M, Buelli S, Pezzotta A, Corna D, Perico L, Tomasoni S, Rottoli D, Rizzo P, Conti D, Thurman JM, Remuzzi G, Zoja C, Morigi M: Shiga toxin promotes podocyte injury in experimental hemolytic uremic syndrome via activation of the alternative pathway of complement. J Am Soc Nephrol 2014;25:1786-1798.

32 Zykova SN, Tveita AA, Rekvig OP: Renal DNase 1 enzyme activity and protein expression is selectively shut down in murine and human membranoproliferative lupus nephritis. PLoS One 2010;5:e12096. 